\title{
Physicochemical interactions at the dentin/adhesive interface using FTIR chemical imaging
}

\section{Paulette Spencer}

University of Missouri-Kansas City

School of Dentistry

Department of Pediatric Dentistry

Kansas City, Missouri 64108

\section{Yong Wang}

\section{J. Lawrence Katz}

University of Missouri-Kansas City

School of Dentistry

Department of Oral Biology

Kansas City, Missouri 64108

\section{Anil Misra}

University of Missouri-Kansas City School of Computing and Engineering

Kansas City, Missouri 64108

\begin{abstract}
To date, much of our understanding of dentin bonding has been based on investigations performed on sound, healthy dentin. This is not the substrate generally encountered in clinical practice, rather dentists must frequently bond to caries-affected dentin. Because of the extreme complexity and variability of the caries-affected dentin substrate, conventional characterization techniques do not provide adequate information for defining those factors that impact bond formation. Using Fourier-transform infrared imaging, we characterized the inhomogeneities and compositional differences across the length and breadth of the caries-affected dentin/adhesive interface. Differences in mineral/matrix ratio, crystallinity, and collagen organization were noted in the comparison of caries-affected and healthy dentin. As compared to healthy dentin, there were striking differences in depth of demineralization, adhesive infiltration, and degree of conversion at the interface with caries-affected dentin. ๑ 2005 Society of PhotoOptical Instrumentation Engineers. [DOI: 10.1117/1.1914844]
\end{abstract}

Keywords: infrared; dentin; adhesive; caries; interface; tooth.

Paper SS04156 received Aug. 4, 2004; revised manuscript received Oct. 20, 2004; accepted for publication Nov. 28, 2004; published online May 24, 2005.

\section{Introduction}

Replacement of failed restorations accounts for nearly $75 \%$ of all operative dentistry ${ }^{1,2}$ and this emphasis on replacement therapy is only expected to grow as the public's concern about mercury release from dental amalgam forces dentists to select alternative restorative materials, e.g., composite resin. The failure rate for large to moderate posterior composite restorations can be $2-3$ times that of high copper amalgam. ${ }^{3}$ The higher failure rate means increased frequency of replacement with loss of additional tooth structure. ${ }^{4,5}$ At 9.6 years, $30 \%$ of the class II composites in one clinical study had failed and half of these failures were due to recurrent caries at the gingival margin. ${ }^{6}$ Generally there is very little enamel available for bonding at this site and thus, the integrity of the gingival margin depends on the bond formed with dentin. ${ }^{7}$ Failure of the dentin bond allows bacterial enzymes, oral fluids, and bacteria to infiltrate the spaces between the tooth and composite, undermining the restoration and leading to recurrent caries, hypersensitivity, and pulpal inflammation. ${ }^{8-10}$

Current adhesives bond to dentin via a hybrid layer (HL). ${ }^{11,12}$ The HL is formed when adhesive resin penetrates the demineralized dentin, infiltrating the exposed collagen to create a continuous integrated collagen/resin network that bonds the bulk adhesive to the intact dentin. ${ }^{13-17}$ The durability of the dentin/adhesive $(d / a)$ bond is directly related to the quality of the HL that connects the bulk adhesive to the subjacent, intact dentin.

Much of our understanding of dentin bonding has been based on results from in vitro bond strength studies performed

Address all correspondence to Dr. Paulette Spencer. Fax: 816-235-5524; e-mail: spencerp@umkc.edu on sound, flat polished healthy dentin. Although the results are useful when comparing bonding systems, healthy dentin is not the substrate most frequently encountered in clinical situations. Instead, clinicians usually must bond adhesives to caries-affected $(c-a)$ dentin or abraded-sclerotic dentin.

Results from both morphologic and bond strength studies suggest that the characteristics of the substrate directly impact the bond formed at the dentin/adhesive interface. ${ }^{18,19}$ For example, using the same material on both healthy and affected (caries-affected or sclerotic) dentin previous authors have reported nearly a 30\%-40\% drop in bond strength with both affected dentin substrates. ${ }^{20,21}$ The hybrid layers formed on $c$ - $a$ dentin were thicker than those formed on healthy dentin. Presumably, the thicker hybrid layer is related to the fact that $c-a$ dentin is already partially demineralized and offers a more porous substrate for acid etching than healthy dentin. These results, which describe distinct differences between bonding characteristics to healthy and caries-affected dentin, highlight the need for investigations on clinically relevant substrates as part of our efforts to develop adhesives that provide durable function under clinical conditions. Our understanding of adhesive bonding to clinically relevant substrates such as caries-affected dentin is very limited. The factors and mechanisms involved in the very low bond strengths to caries-affected dentin remain unclear.

Fourier-transform infrared imaging (FTIRI) represents the combination of spectroscopy with microscopy and allows the collection of molecular information at the microscopic level. The Fourier-transform infrared (FTIR) images represent the inherent contrast associated with the unique chemical bonds

1083-3668/2005/\$22.00 ㄷ 2005 SPIE 
of the components within the analyzed sample. A chemical image of each component is created by plotting the unique frequencies as a function of spatial position and spectral intensity. A color scheme can be applied to the intensity values to permit the creation of a "false-color composite" image. In this study, FTIRI was used to characterize the interface structure of adhesive bonded to caries-affected and healthy dentin and provide insight into those factors that impact dentin bonding to clinically relevant substrates.

\section{Materials and Methods Specimen Preparation}

Three extracted unerupted human third molars and three extracted human molars with coronal caries were collected from the Oral Surgery Clinic at the University of Missouri-Kansas City (UMKC) School of Dentistry. The teeth were collected after the patient's informed consent was obtained under a protocol approved by the UMKC adult health sciences institutional review board. Following extraction the teeth were placed in separate vials containing $0.9 \%$ normal saline and $0.002 \%$ sodium azide and stored at $4{ }^{\circ} \mathrm{C}$. For healthy teeth, initial specimen preparation proceeded as follows: the occlusal one-third of the crown was sectioned perpendicular to the long axis of the tooth by means of a water-cooled low speed diamond saw (Buehler, Lake Bluff, IL). The exposed dentin surfaces were inspected with a microscope to ensure that no enamel remained. A uniform smear layer was created by abrading the exposed dentin surface with 600 grit $\mathrm{SiC}$ under water for $30 \mathrm{~s}^{22,23}$

The preparation of $c-a$ dentin specimens followed the technique described by Nakajima et al. ${ }^{19,24}$ Characteristically, carious dentin is described as consisting of infected and affected layers. The affected layer is generally not removed during treatment. The carious material could be identified by visual inspection following staining with a caries detector solution. The occlusal surface was ground perpendicular to the long axis of the tooth until a flat surface composed of the carious lesion surrounded by normal dentin is exposed.

Prepared healthy dentin as well as prepared $c-a$ dentin substrates were selected for treatment with a current commercial dentin adhesive Single Bond (3M/ESPE Dental Products, St. Paul, MN). The application of the adhesive followed the manufacturer's instructions. The different dentin surfaces were etched for $15 \mathrm{~s}$ with $35 \%$ phosphoric acid. After acid etching, the teeth were rinsed with water for $10 \mathrm{~s}$ and blotted dry, leaving the dentin surface moist. Two consecutive coats of Single Bond were applied with a fully saturated brush. The surface was gently dried for $5 \mathrm{~s}$ and light cured for $20 \mathrm{~s}$. These specimens were stored for a minimum of $24 \mathrm{~h}$ in $\mathrm{H}_{2} \mathrm{O}$ at $37^{\circ} \mathrm{C}$ before proceeding with the sectioning. The treated dentin surfaces were cut perpendicular to the bonded surface at 2 mm increments by means of a $\mathrm{H}_{2} \mathrm{O}$ cooled low-speed diamond saw. The specimens were then cut at a depth of $\sim 2 \mathrm{~mm}$ below the interface to create a number of resin bonded beams or slabs. The final dimensions of these slabs were $10 \times 2$ $\times 2 \mathrm{~mm}$.

\section{Sectioning}

The rectangular beams were mounted on a methacrylate support with cyanoacrylate adhesive and $3-\mu$ m-thick sections were cut perpendicular to the adhesive-dentin face of the beam using a tungsten carbide knife mounted on a Leica Polycut $\mathrm{S}$ microtome (Leica, Deerfield IL). The knife edge and beams were moistened with distilled water while cutting. As each single section is cut, it is allowed to slide onto the knife, the microtome is stopped, and the thin section was collected on a $13 \mathrm{~mm}$ diameter, 2-mm-thick barium fluoride disk with the aid of a fine paintbrush. Most important for high quality image is the flatness of the sections at the end of procedure. For this reason, sections were covered by another $\mathrm{BF}_{2}$ disk to keep them flat. These sections were placed directly on the motorized stage for FTIR microspectroscopic and imaging analyses.

\section{FTIR Microspectroscopic Imaging Analysis}

FTIR microspectroscopic and imaging analyses were completed using the Spectrum Spotlight FTIR imaging system (Perkin Elmer) with both single point and imaging mode. The liquid nitrogen cooled detector incorporates a narrow band mercury-cadmium-telluride (MCT) array detector and single point medium band MCT detector on a single substrate. It offers the wavelength range from 7800 down to $720 \mathrm{~cm}^{-1}$, supplying the mineral fingerprint detail missing from all other FTIR imaging systems. This system provides 25 and $6.25 \mu \mathrm{m}$ pixel resolution, and could generate chemical image size up to $1.64 \mathrm{~cm}^{2}$. Images were scanned between 4000 and $720 \mathrm{~cm}^{-1}$ at $4 \mathrm{~cm}^{-1}$ spectral resolution, with two scans per pixel. Image size was $\sim 300$ by $\sim 300 \mu \mathrm{m}$, using $6.25 \mu \mathrm{m}$ pixel resolution. Approximately 2600 spectra were acquired in less than 5 min. An atmosphere correction was applied to the raw image, subtracting the contribution of atmosphere absorbance, i.e., water vapor and carbon dioxide. Images were created using Spectrum Spotlight software. In some cases, individual spectra were extracted from selected areas for more detailed analysis. The amide $\mathrm{I}$ band and the $\nu_{1} \nu_{3} \mathrm{PO}_{4}$ domain between 1200 and $900 \mathrm{~cm}^{-1}$ were deconvoluted with Spectrum 3.3 software (Perkin Elmer). The deconvolution parameters were: gamma at 1.2 and no smoothing (length $=0)$.

\section{Results \\ FTIR Spectra of Adhesive and Dentin}

The infrared spectra for Single Bond adhesive as well as healthy and carious dentin are presented in Figs. 1 and 2, respectively. The tentative assignments of the spectral features for adhesive and dentin are presented in Table 1. For Single Bond, the more intense bands occur at $1720 \mathrm{~cm}^{-1}$ (carbonyl), $1457 \mathrm{~cm}^{-1} \quad\left(\mathrm{CH}_{2} \mathrm{CH}_{3}\right) \quad 1165 \mathrm{~cm}^{-1} \quad(\mathrm{C}-\mathrm{O}$ stretch$)$, and $832 \mathrm{~cm}^{-1}(\mathrm{C}-\mathrm{C}-\mathrm{O})$. These bands are associated with methacrylate monomers in adhesive liquid. Representative healthy and caries-affected dentin spectra are presented in Fig. 2. All spectra were recorded from 720 to $2000 \mathrm{~cm}^{-1}$, which spans the fingerprint region associated with collagen and mineral. The principal IR active bands associated with dentin have been well characterized. The most intense band at $900-1100 \mathrm{~cm}^{-1}$ range is associated with the inorganic component. Collagen features are present at $1650 \mathrm{~cm}^{-1}$ (amide I), $1550 \mathrm{~cm}^{-1}$ (amide II), $1450 \mathrm{~cm}^{-1}(\mathrm{CH})$, and $1240 \mathrm{~cm}^{-1}$ (amide III). The differences in spectra are clearly noticed in both the protein amide bands and $\nu_{1} \nu_{3} \mathrm{PO}_{4}$ bands. The ap- 


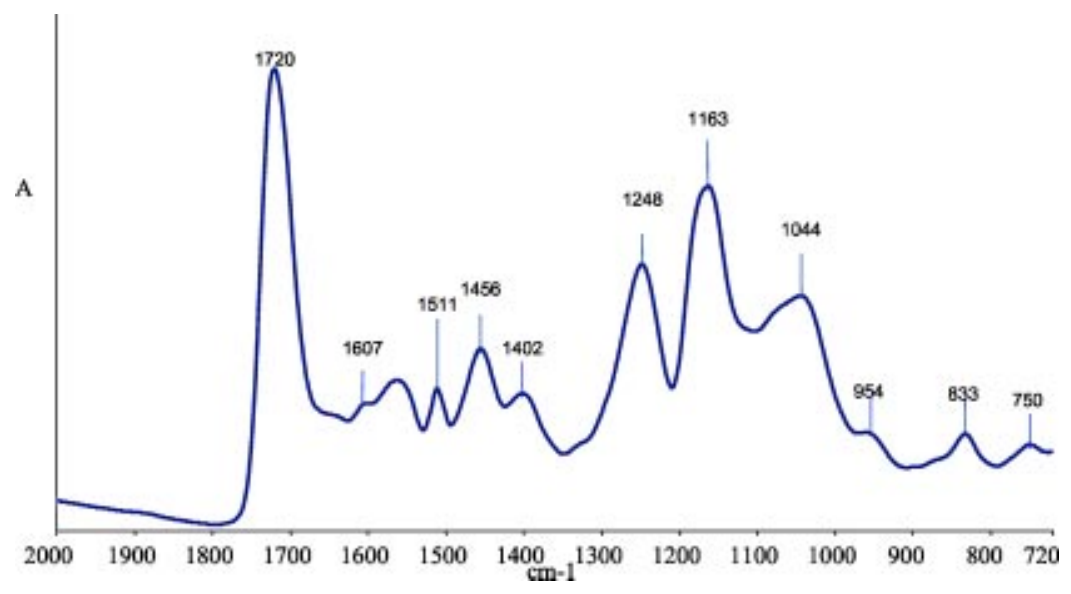

Fig. 1 Infrared spectrum of the single bond adhesive in the region $2000-720 \mathrm{~cm}^{-1}$.

parent feature is the mineral/collagen area ratio that is significantly smaller for caries-affected dentin as compared to healthy dentin.

Changes in the spectral features associated with the collagen component are also shown in Figs. 2 and 3(a). Amide bands are thought to be representative of protein conformation. The amide I band is shifted from 1655 to $1638 \mathrm{~cm}^{-1}$ and amide II band also is shifted from 1553 to $1546 \mathrm{~cm}^{-1}$ (Fig. $2)$. In a comparison of this reported region in the deconvoluted spectra of healthy and $c$ - $a$ dentin [Fig. 3(a)], the relative intensity of this amide I band has changed dramatically and lost its asymmetrical structure in $c-a$ dentin. The amide I ( $\mathrm{C}=\mathrm{O}$ stretching vibration) mode, with three bands at 1694 , $1660,1634 \mathrm{~cm}^{-1}$, is sensitive to the molecular conformation of the polypeptide chains. These spectral changes indicate that the ordered collagen structure became disorganized and denatured. The broadening and shift to low frequencies of this band could be caused by a loosening of the collagen fibril structure from water absorption. ${ }^{25}$
The $\nu_{1} \nu_{3} \mathrm{PO}_{4}$ domain between 1150 and $930 \mathrm{~cm}^{-1}$ is explored to obtain data essentially on mineral crystallinity [Figs. 2 and 3(b)]. The $\nu_{1} \nu_{3} \mathrm{PO}_{4}$ domain is shifted from $1040 \mathrm{~cm}^{-1}$ in the healthy dentin to $1014 \mathrm{~cm}^{-1}$ in the $c-a$ dentin. In the $c$ - $a$ dentin spectrum, the greater broadness of the $\nu_{1} \nu_{3} \mathrm{PO}_{4}$ domain band and shift to low frequencies indicate that the mineral structure and crystallinity alter dramatically (Fig. 2). The healthy and $c-a$ dentin shows the different $\nu_{1} \nu_{3} \mathrm{PO}_{4}$ deconvolution patterns [Fig. 3(b)]. The $1032 \mathrm{~cm}^{-1}$ is representative of stoichiometric apatites and the $1020 \mathrm{~cm}^{-1}$ of less crystalline apatites. The $1020 / 1030 \mathrm{~cm}^{-1}$ intensity ratio is frequently used for measuring degree of crystallinity. In the $c$ - $a$ dentin, the most intense band is shifted to $1011 \mathrm{~cm}^{-1}$, indicating the decreased crystallinity. The bands located between 1050 and $1100 \mathrm{~cm}^{-1}(1060,1070,1095$, $1100 \mathrm{~cm}^{-1}$ due to different $\mathrm{PO}_{4}^{3-}$ environments) also shows some differences between healthy and $c$ - $a$ dentin.

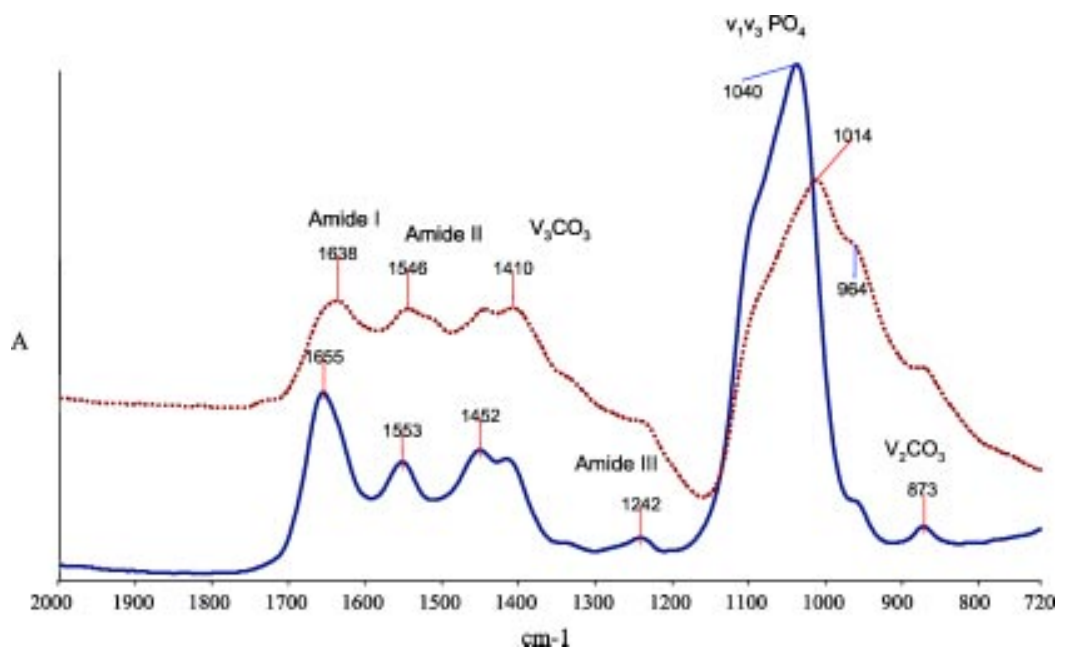

Fig. 2 Infrared spectra of healthy dentin (solid line) and caries-affected dentin (dotted line) in the region of $2000-720 \mathrm{~cm}^{-1}$. 
Table 1 Infrared spectroscopic band assignments for the Single Bond adhesive and dentin.

\begin{tabular}{|c|c|}
\hline Wavenumber range $\left(\mathrm{cm}^{-1}\right)$ & Assignment \\
\hline \multicolumn{2}{|l|}{ Adhesive } \\
\hline $1690-1740$ & carbonyl $\mathrm{C}=\mathrm{O}$ \\
\hline $1635-1645$ & $\mathrm{C}=\mathrm{C}$ \\
\hline $1605-1609$ & phenyl $\mathrm{C}=\mathrm{C}$ \\
\hline $1445-1457$ & $\mathrm{CH}_{2}$ \\
\hline $1402-1406$ & $=\mathrm{CH}_{2}$ deformation \\
\hline $1165-1180$ & $\mathrm{C}-\mathrm{O}$ stretch \\
\hline $830-836$ & $\mathrm{C}-\mathrm{C}-\mathrm{O}$ stretch \\
\hline \multicolumn{2}{|l|}{ Dentin } \\
\hline $1596-1715$ & Amide I \\
\hline $1540-1582$ & Amide ॥ \\
\hline $1445-1456$ & $\mathrm{CH}_{2}$ \\
\hline 1145 & $\mathrm{HPO}_{4}^{2-}$ \\
\hline $1100-1109$ & $\nu_{3} \mathrm{PO}_{4}^{3-}$ \\
\hline $1060-1075$ & $\nu_{3} \mathrm{PO}_{4}^{3-}$ in poorly crystalline apatites \\
\hline 1030 & $\nu_{3} \mathrm{PO}_{4}^{3-}$ in stoichiometric apatites \\
\hline 1020 & $\nu_{3} \mathrm{PO}_{4}^{3-}$ in poorly crystalline apatites \\
\hline $957-961$ & $\nu_{1} \mathrm{PO}_{4}^{3-}$ \\
\hline $860-890$ & $\mathrm{CO}_{3}^{2-}$ \\
\hline
\end{tabular}

\section{Two-Dimensional FTIR Imaging of the Dentin/}

\section{Adhesive Interface}

By combining spectroscopy with microscopy, molecular information can be obtained with great spatial resolution at the microscopic level. Specimens can be analyzed directly, in air, at room temperature and without destroying the specimen. In this study, the dentin/adhesive interface was analyzed by FTIR imaging. Figure 4 presents the visible image of the microtomed healthy dentin/adhesive interface slice. The two layers, i.e., dentin and adhesive are clearly visible, although lacking in detail. Figure 5(a) shows the total absorbance image of the above healthy dentin/adhesive interface collected from a $200 \times 150 \mu \mathrm{m}^{2}$ area. While there is no chemical information in the full absorbance image, it is a spatial representation of the energy impinging on the detectors. It is noted, however, that some additional features are observed, indicating stronger absorbance (less energy impinging the detectors) in the bottom half of the image. Inspection of Figs. 5(b)-5(d) reveals clear delineation between layers. Images of three spectral parameters $\left(1721,1658\right.$, and $\left.1027 \mathrm{~cm}^{-1}\right)$ provide information about the spatial distribution of the adhesive, collagen and mineral (apatite) components across the healthy $d / a$ interface.
Investigation of all of the images indicates that the interface is not a thin slice through the images. Its width appears to indicate an interaction between the adhesive and dentin layers, which is not obvious from observation of the visible image (Fig. 4). The interfacial width of $1027 \mathrm{~cm}^{-1}$ image appears to be bigger than that of $1721 \mathrm{~cm}^{-1}$ image. This indicates that the width of demineralized dentin layer is much deeper than that of the adhesive could penetrate, which is consistent with our previous micro-Raman results showing the gradient penetration of adhesive into the demineralized dentin layer. ${ }^{17,26}$

Since these images contain thousands of very high quality spectra at a pixel resolution of $6.25 \mu \mathrm{m}$, the relative composition and other chemical information can be determined across the length and breadth of the $d / a$ interface. As an example, a series of mapping spectra across the healthy dentin/ adhesive interface [the position was represented by the dotted line in Fig. 5(d)] are shown in Fig. 6. The chemical characteristics of the two layers are apparent in the spectra. The three bottom spectra were acquired from pure adhesive, a methacrylate-based polymer. Vibrational bands associated with both adhesive and dentin components are noted in the fourth spectrum. The spectral intensity of the $\nu_{1} \nu_{3} \mathrm{PO} 4$ domain increase gradually as a function of depth. The high spectral intensity of the $\nu_{1} \nu_{3}$ PO4 domain in the top spectrum indicates mineralized dentin. Spectral features associated with both adhesive $\left(1721 \mathrm{~cm}^{-1}\right)$ and collagen $\left(1658 \mathrm{~cm}^{-1}\right)$ appear in several spectra across the interface. This indicates that within the limits of resolution of this technique, the adhesive resin has penetrated into the dentin matrix, although the width of the interface layer could be overexaggerated due to the low spatial resolution of FTIR.

Representative microspectroscopic images across the caries-affected dentin/adhesive interface are shown in Fig. 7. Figure 7(a) shows the total absorbance image of the $c-a$ dentin/adhesive interface collected from a $300 \times 280 \mu \mathrm{m}^{2}$ area. While there is no chemical information in the total absorbance image, it is noted, however, that a more complicated absorbance pattern is observed in this image. As shown in Figs. 7(b)-7(d), three images of spectral parameters (1721, 1658 , and $1027 \mathrm{~cm}^{-1}$ ) were generated, representing the spatial distribution of the adhesive, collagen and mineral (apatite) components across the $c-a d / a$ interface. It is noticed that the images of adhesive, collagen and mineral reveal very complex patterns in the interface. As compared to the healthy dentin/ adhesive interface, the width of the interface is much bigger, and also the interface is not a uniform layer; it appears to intrude into either layer.

Due to the wide interface, two series of mapping spectra across the $c$ - $a$ dentin/adhesive interface are shown in Figs. 8 (a) and 8(b) separately [the position was represented by the dotted lines 1 and 2 in Fig. 7(d), respectively]. As shown in Fig. 8(a), the amide I band is gradually shifted from 1656 to $1637 \mathrm{~cm}^{-1}$ across from healthy dentin to caries-affected dentin. This frequency shift indicates that the ordered collagen structure in healthy dentin changes to the disorganized and denatured structure in the $c$ - $a$ dentin. Similarly, the $\nu_{1} \nu_{3} \mathrm{PO}_{4}$ domain becomes broad and is gradually shifted from 1038 to $1015 \mathrm{~cm}^{-1}$ across from the healthy dentin to the $c-a$ dentin. The broadening of the $\nu_{1} \nu_{3} \mathrm{PO}_{4}$ domain band and gradual shift to low frequencies indicate that the mineral structure 


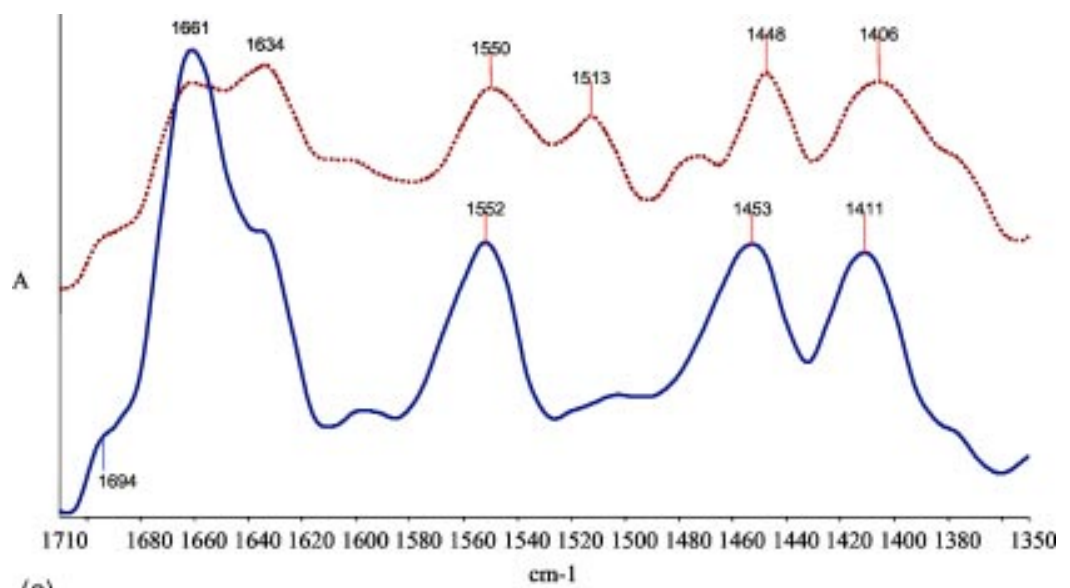

(a)

$$
\mathrm{cm}-1
$$

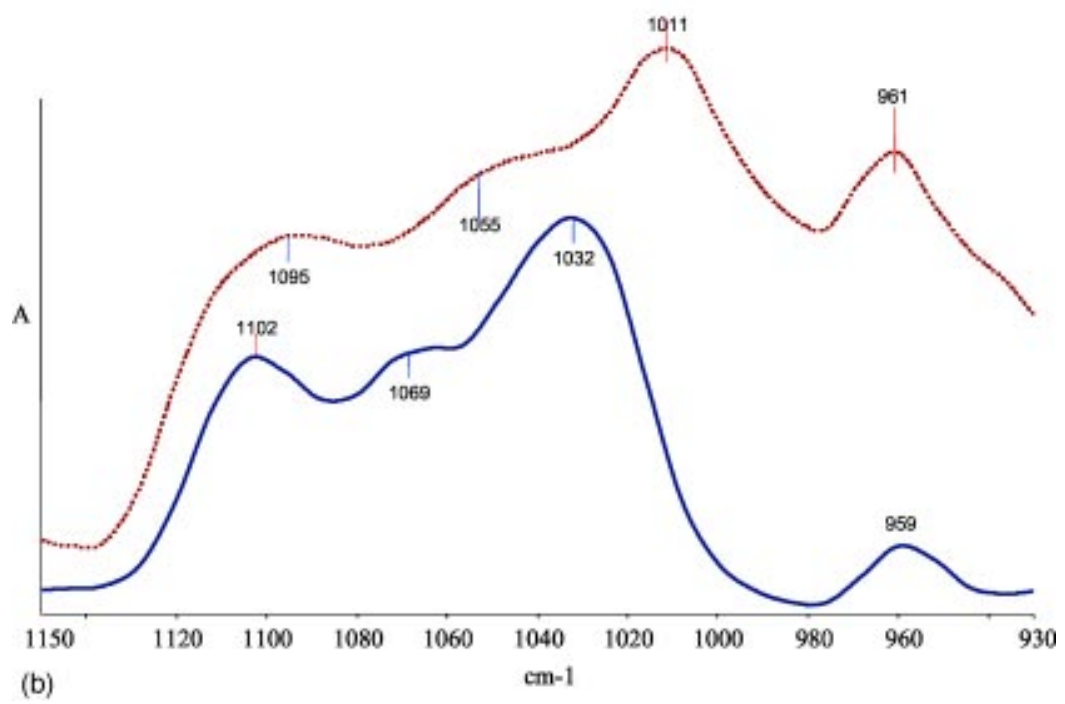

Fig. 3 Deconvoluted spectra of healthy dentin (solid line) and caries-affected dentin (dotted line). (a) In the region of $1710-1350 \mathrm{~cm}^{-1}$; (b) $\nu_{1} \nu_{3}$ $\mathrm{PO}_{4}$ domain between 1150 and $930 \mathrm{~cm}^{-1}$.

undergoes dramatic changes when the dentin becomes caries affected. The relative intensity of the $\nu_{1} \nu_{3} \mathrm{PO}_{4}$ domain also gradually decreased, indicating the caries-affected dentin has less mineral content as compared to the healthy dentin. A series of mapping spectra across the $c$ - $a$ dentin/adhesive interface are presented in Fig. 8(b). Spectral features associated with both adhesive $\left(1721 \mathrm{~cm}^{-1}\right)$ and collagen $\left(1658 \mathrm{~cm}^{-1}\right)$ are observed in several spectra across the interface. This indicates that the adhesive resin has penetrated into the demineralized dentin layer.

Representative microspectroscopic images collected from the adhesive region close to the healthy dentin and cariesaffected dentin surface are presented in Figs. 9 and 10, respectively. Images were generated based on the band ratios of $1640(\mathrm{C}=\mathrm{C}) / 1606$ (phenyl), which are used to measure the degree of double bond conversion (DC) in the light-cured adhesive resin. Red-yellow represents the highest relative intensity (lowest DC) while blue represents the lowest (highest DC). The image representing distribution of the degree of conversion in the adhesive layer on the top of the healthy dentin surface is shown in Figs. 9 and 10. Two spectra corresponding to the demarcations in the image were also shown.
The DC of adhesive on the top of $c$ - $a$ dentin surface is lower as compared to that of the adhesive layer on healthy dentin surface. The DC of resin monomer differs substantially, and overall, the DC is lower in the region close to dentin surface. The differences in resin monomer conversion are likely due to outward water from dentin tubules as it interacts with the wet demineralized dentin matrix. Such survey maps of molecular information provide a reliable and powerful means of identifying resin monomer conversion distribution and flaws or defects in the pattern of adhesive/dentin interface.

\section{Discussion}

Understanding the structure of clinically relevant dentin substrates is vital to the development of improved dentin bonding agents and ultimately, composite restorations. However, the complexity of the biopolymer that forms at the interface with clinically relevant substrates presents a formidable challenge. Conventional characterization techniques, e.g., bond strength measurements, cannot adequately define the factors that lead to crack initiation or the elements that affect premature failure at the complex interface that forms between synthetic adhe- 


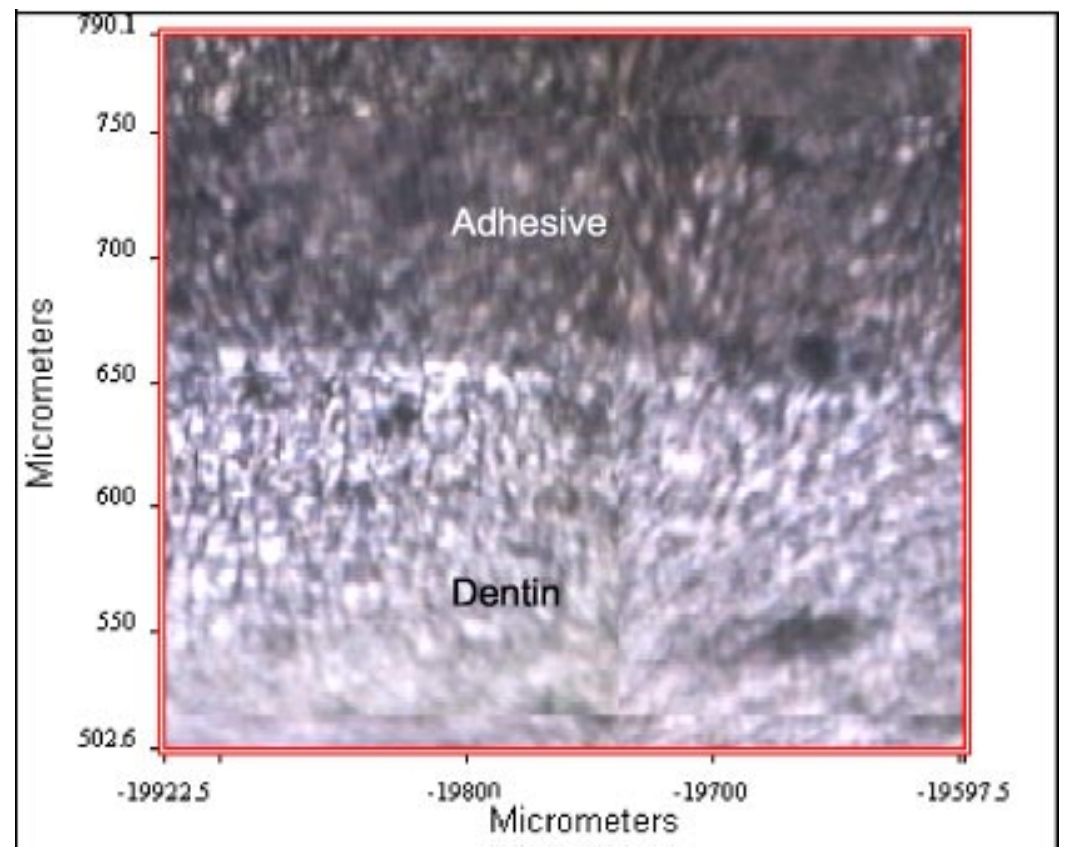

Fig. 4 Visible image of the microtomed slice of the dentin-adhesive interface. The regions associated with the adhesive and dentin are indicated on the figure.

Total Ab

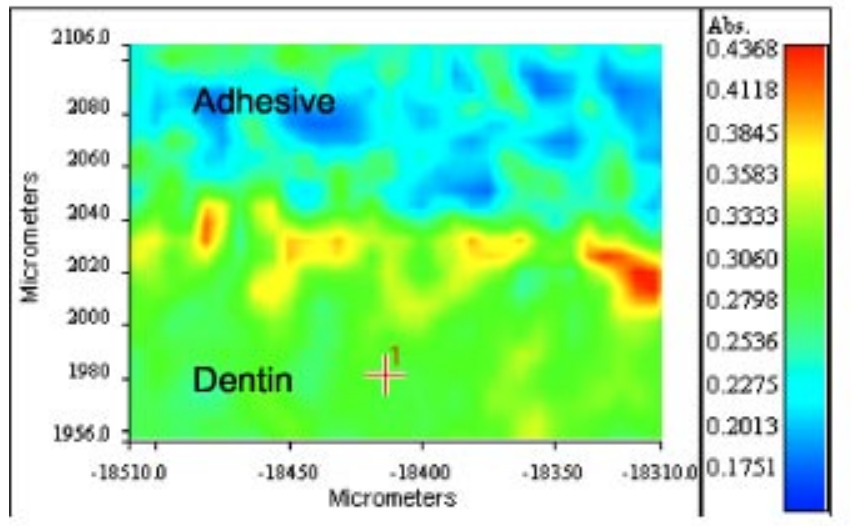

1658

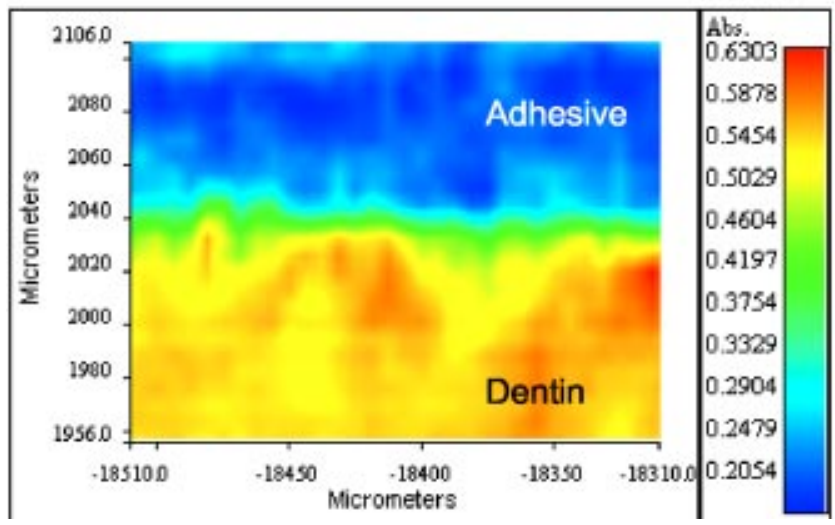

1721

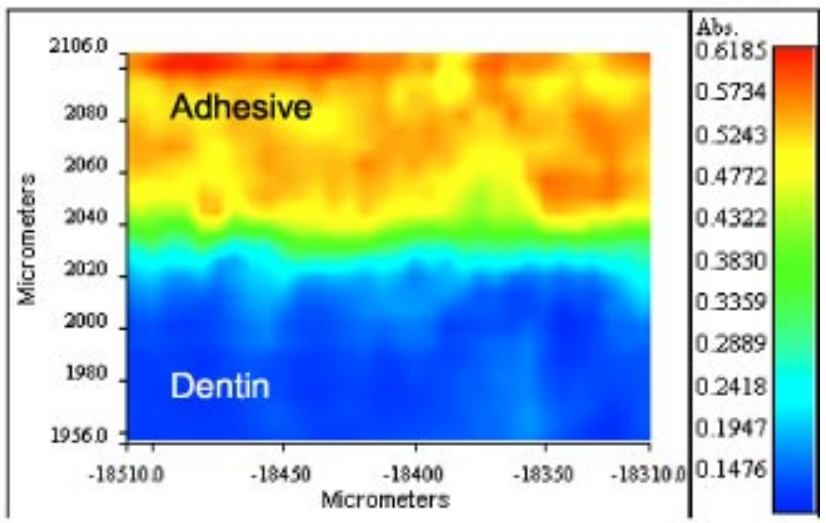

\section{7}

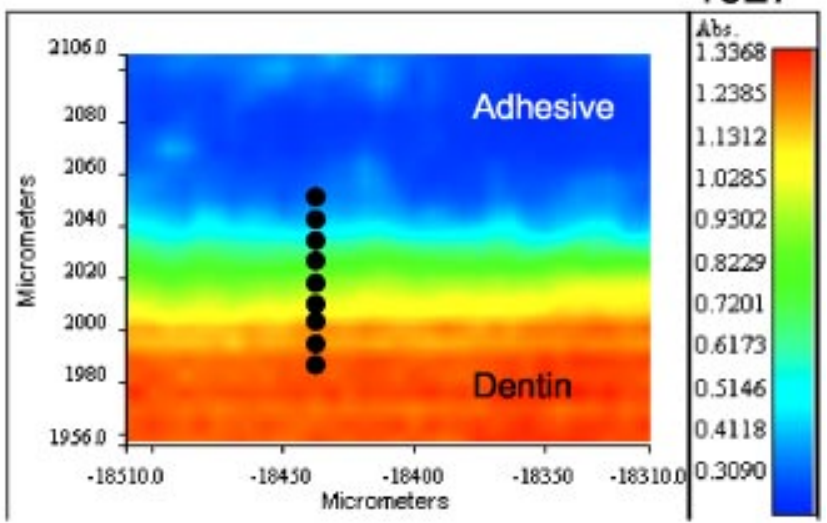

Fig. 5 Infrared spectroscopic images of the healthy dentin-adhesive interface (a) total absorbance image; (b) $1721 \mathrm{~cm}^{-1}$ image (carbonyl); (c) $1658 \mathrm{~cm}^{-1}$ image (amide I); (d) $1027 \mathrm{~cm}^{-1}$ image (phosphate). 


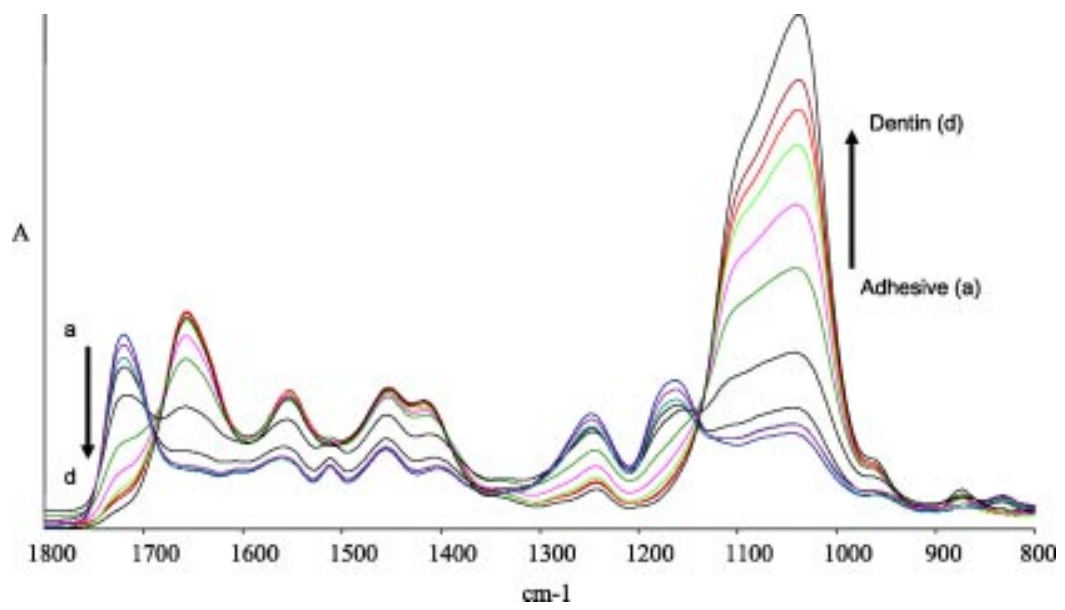

Fig. 6 Mapping spectra across the healthy dentin-adhesive interface. These spectra are associated with the black dots noted across the dentinadhesive interface in Fig. 5(d).

Total Ab
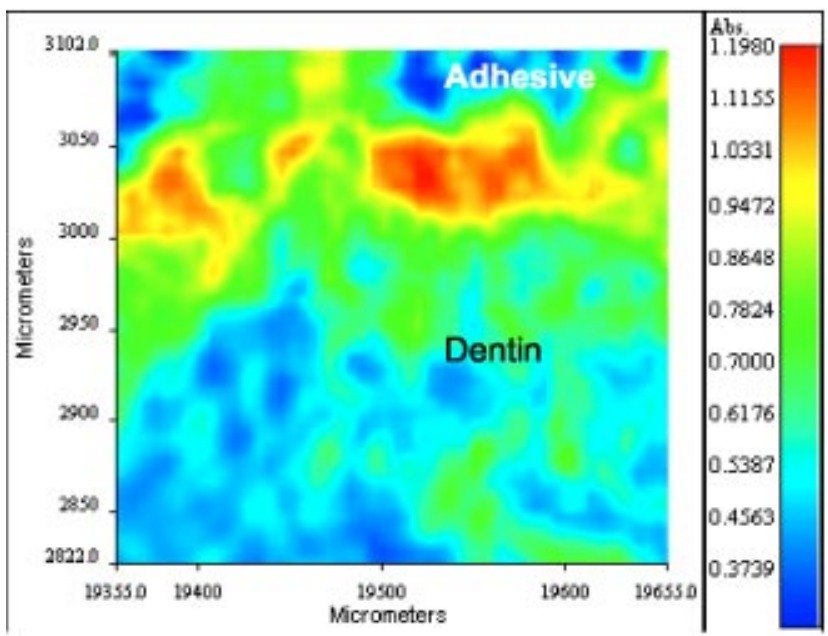

1643

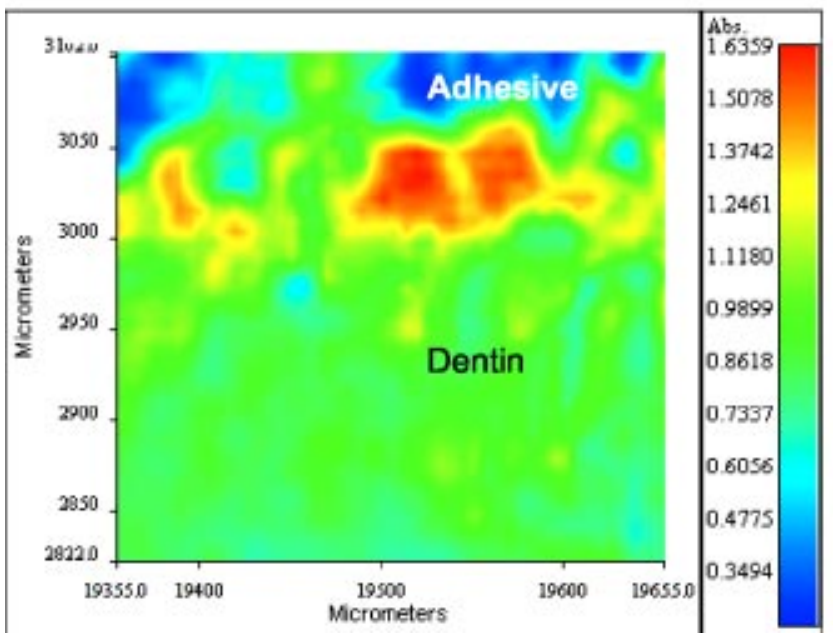

1721

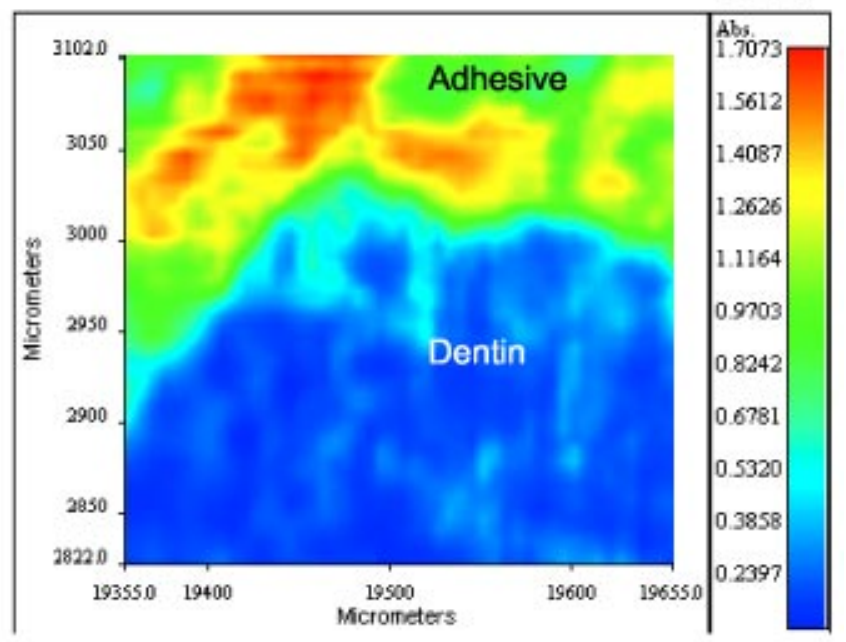

1027

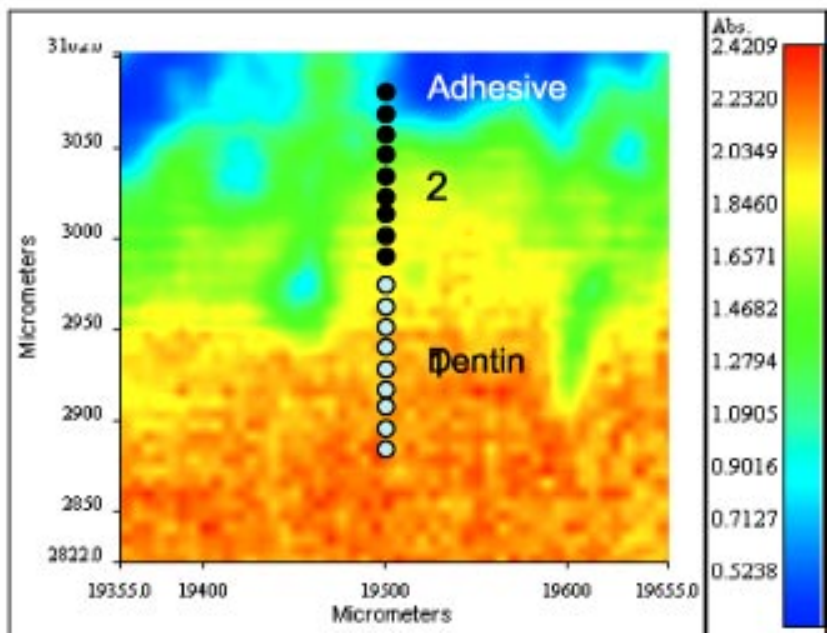

Fig. 7 Infrared spectroscopic images of the caries-affected dentin-adhesive interface (a) total absorbance image; (b) $1721 \mathrm{~cm}^{-1}$ image (carbonyl); (c) $1658 \mathrm{~cm}^{-1}$ image (amide I); (d) $1027 \mathrm{~cm}^{-1}$ image (phosphate). 


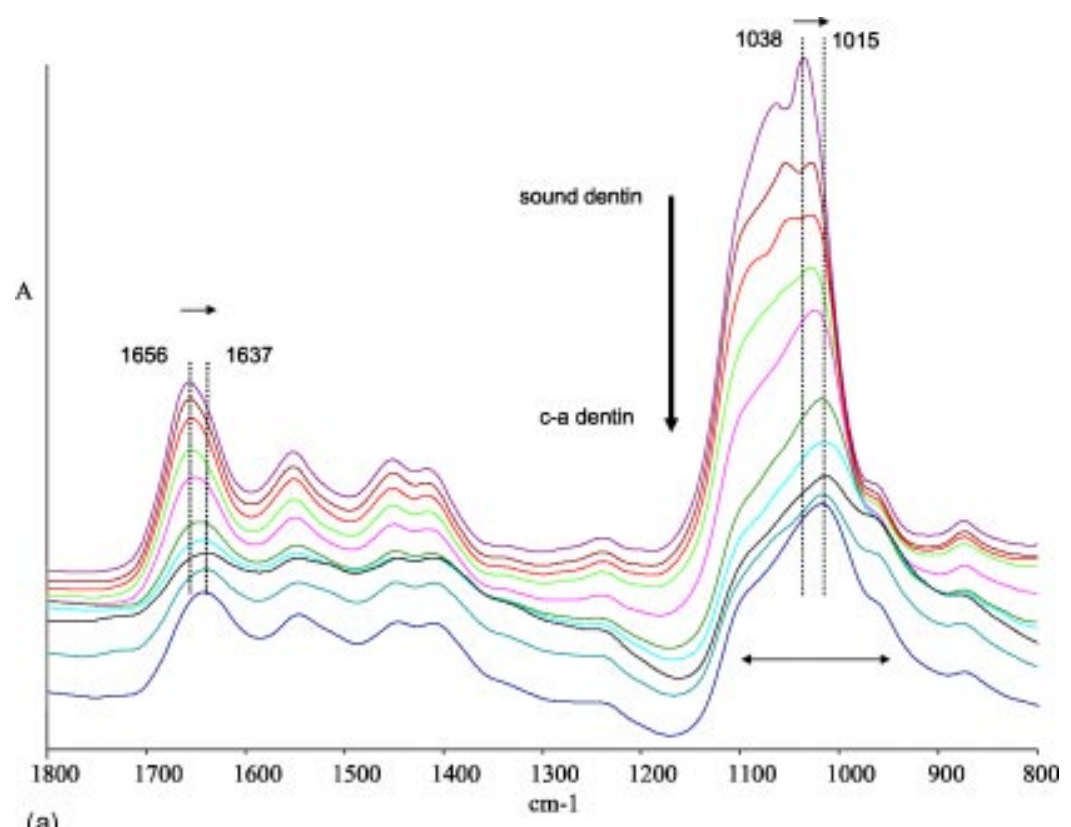

(a)

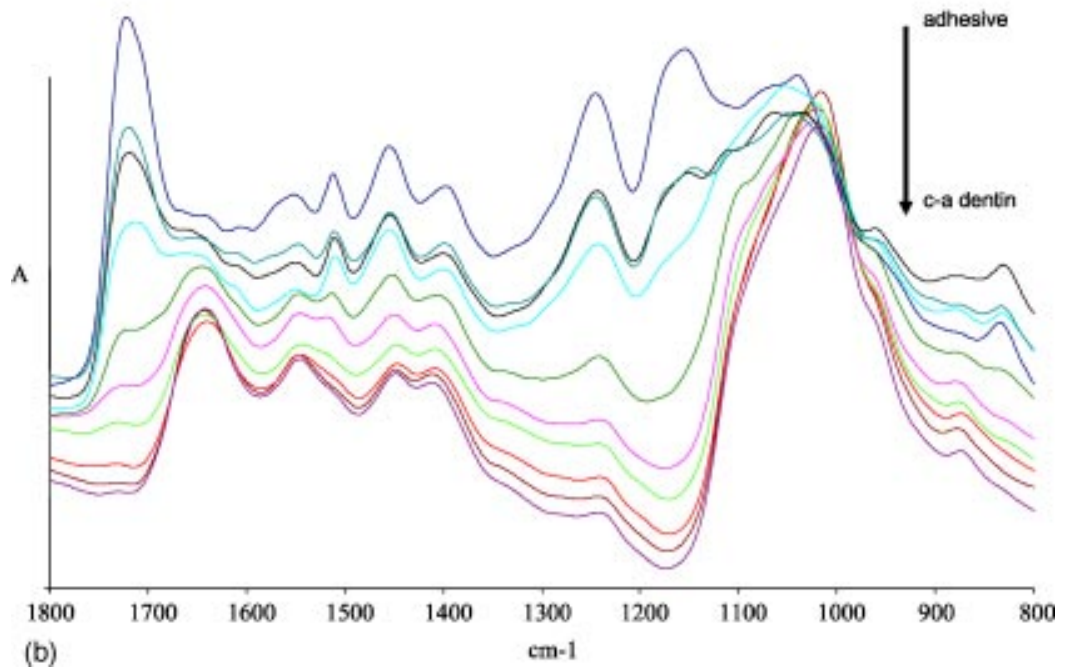

Fig. 8 Mapping spectra across the caries-affected dentin-adhesive interface. (a) These spectra are associated with the black dots identified as 1 in the infrared spectroscopic image shown in Fig. 7(d). (b) These spectra are associated with the black dots identified as 2 in the infrared spectroscopic image shown in Fig. 7(d).

sive and caries-affected dentin. As an example, testing methods that measure fracture resistance of bulk $d / a$ specimens before and after water immersion are not sensitive enough to identify interfacial defects where aqueous degradation begins. $^{27}$ Analytical techniques that allow direct nondestructive, in situ detection of the variable composition and inhomogeneity across the length and breadth of the interface are required.

By combining spectroscopy (infrared and Raman) with microscopy, molecular information can be obtained with great spatial resolution at the microscopic level. FTIR microspectroscopy can be used to determine the relative composition, degree of cure and homogeneity across the length and breadth of the $d / a$ interface. The spotlight imaging system displays false color IR images almost as fast as an optical microscope presents visible images thus, spectroscopic survey maps of the $d / a$ interface can be collected in a very time and laborefficient manner.

In comparing the results from the health and cariesaffected dentin, striking differences are noted in the spectra collected from these substrates. Features associated with the mineral component are broadened and shifted to lower frequencies in the caries-affected specimens; these changes indicate poorly crystalline apatite in the $c$ - $a$ dentin [Figs. 2 and 3(b)]. Similar differences are noted in the spectral features associated with the organic component. The amide I region shows a dramatic loss of structure and intensity in the spectra recorded from the $c$ - $a$ dentin, indicating disorganized and denatured collagen in these specimens (Fig. 3). Of particular note is the substantially smaller mineral/collagen area ratio in 

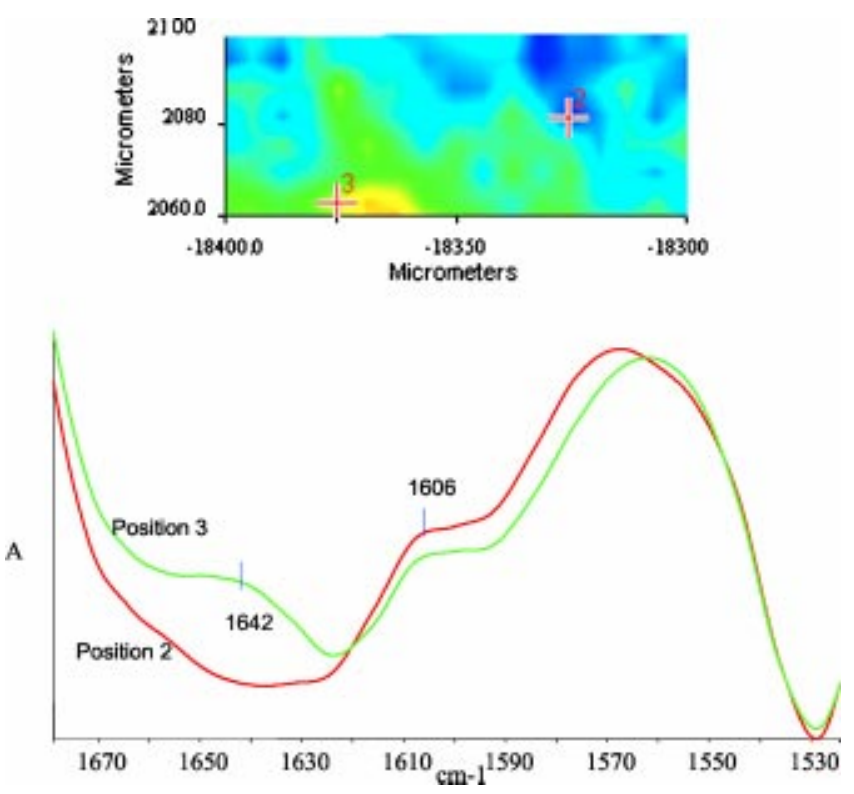

Fig. 9 Infrared spectroscopic image of the spatial distribution of double bond conversion in adhesive layer of healthy dentin-adhesive interface.

the $c$ - $a$ dentin. In concert the spectral differences reflect a dramatic alteration in the structure of $c-a$ dentin including a loss of crystallinity in the mineral, reduced mineral content and loss of organization in the collagen [Figs. 2, 3(a), and 3(b)].

The FTIRI data provided visualization of the spatial distribution of the components across the length and breadth of the dentin/adhesive interfaces. The chemical images are constructed by plotting the intensity for an IR band that corresponds to a selected component. A false-color composite image is created by applying a color scheme to the intensity values. As shown in Figs. 5 and 7, images recorded at 1721,

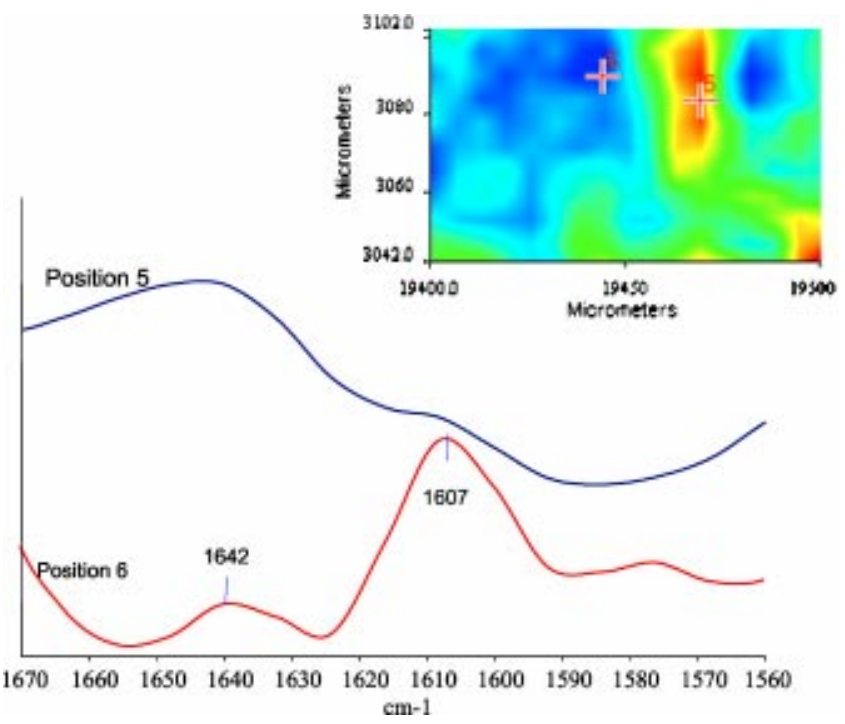

Fig. 10 Infrared spectroscopic image of the spatial distribution of double bond conversion in the adhesive layer of caries affected dentin-adhesive interface.
1658 , and $1027 \mathrm{~cm}^{-1}$ provide information about the spatial distribution of adhesive, protein, and mineral, respectively. The variability and complexity of the composition is portrayed clearly in these chemical images. The interfacial chemistry between Single Bond and $c-a$ dentin reflect a highly irregular composition and lack of homogeneity as compared to healthy dentin. The chemical images contain thousands of very high quality spectra at a pixel resolution of $6.25 \mu \mathrm{m}$; further analyses of these spectra reveal detailed information about the substrates and their interaction with the adhesive.

The mapping spectra in Fig. 6 provide information about the depth of demineralization and adhesive infiltration into the prepared healthy dentin substrate. The interfacial width of the image associated with the mineral component $\left(1027 \mathrm{~cm}^{-1}\right)$ appears larger than the interfacial width in the image associated with the adhesive $\left(1721 \mathrm{~cm}^{-1}\right)$. This indicates that the adhesive did not penetrate the width of the demineralized dentin; these results are consistent with our previous investigations using micro-Raman spectroscopy. It is likely that differences in the spatial resolution of the two techniques accounts for some of the quantitative differences in the extent of adhesive infiltration.

In comparing the mapping spectra of the healthy and caries-affected dentin, distinct differences are noted in the demineralization depth and adhesive infiltration (Figs. 6 and 8 ). The width of the interface in the caries-affected dentin is much larger than the healthy dentin specimens. The interfacial structure and compositional differences in the caries-affected dentin are profoundly complex in comparison to healthy dentin. The substantial area of demineralization in the $c-a$ specimens indicates a porous, poorly mineralized structure that was readily penetrated by the acid etchant. The degree of conversion of the adhesive that penetrated the demineralized dentin in the $c-a$ specimens is lower than in the healthy dentin specimens. This difference is likely related to the very wet environment present within the $c$ - $a$ dentin specimens.

Previous results from our laboratory have provided direct spectroscopic evidence of the limitations associated with Single Bond adhesive when applied to wet demineralized dentin substrates. ${ }^{28,29}$ Based on these previous micro-Raman spectroscopic investigations, water within the demineralized dentin matrix led to partitioning of the components such that the photoinitiator was not available to react with one of the phases within the adhesive. Thus, limited monomer/polymer conversion has been reported previously by our group when this adhesive was applied to wet dentin surfaces. The dramatic effect of the wet environment was not, however, as apparent with normal, healthy dentin. In contrast, analysis of the mapping spectra from the $c$ - $a$ dentin/adhesive specimens reveal the profound effect of the increased moisture on monomer/ polymer conversion in this adhesive.

Most current methods of bonding an adhesive to dentin rely on effective adhesive penetration into demineralized dentin to form the resin hybrid layer ${ }^{30,31}$ or resin-dentin interdiffusion zone. ${ }^{32}$ The last term clearly reflects the complexity of this layer; ideally, this layer is a structurally integrated collagen-resin biopolymer hybrid that provides both a continuous and stable link between the bulk adhesive and dentin substrate. However, in vitro and in vivo studies ${ }^{27,33}$ have shown that this objective is not achieved. The resin-collagen 
biopolymer hybrid that forms at the dentin/adhesive interface is instead characteristically porous thus, it can be easily penetrated and potentially degraded by aqueous solutions, oral fluids and/or proteolytic enzymes. ${ }^{27,33}$

Quantitative differences in the distribution, structure and character of the bonding resin, collagen and mineral at the dentin/adhesive interface have been recorded in our laboratory. ${ }^{16,17,34,35}$ The structure of the collagen varies from denatured and disorganized to finally, its native conformational state. The amount and distribution of mineral varies from complete to partial demineralization. The distribution as well as the monomer/polymer conversion of the bonding resin varies as a function of position. Differences in the structure and properties of the dentin substrates are key factors contributing to the complexity and the variability of the collagenresin biopolymer hybrid formed at the dentin/adhesive interface.

FTIR microspectroscopic imaging does not offer the high spatial resolution capabilities of other techniques such as micro-Raman spectroscopy $(\sim 1 \mu \mathrm{m})$. In spite of this limitation, it offers the advantage that IR spectra, with acceptable signal/noise ratio, can be collected from areas measuring several hundred square micrometers in a manner of minutes. Regions of interest can be quickly identified and analyzed using higher spatial resolution techniques. A research protocol has been developed in our laboratory that permits analysis of the same interface specimens using multiple, complementary methods. Separate microtomed sections recovered from the same $d / a$ interface slab are prepared for FTIR imaging and light microscopic examination using a novel staining technique. ${ }^{16,17,32,33}$ These same $d / a$ interface slabs are also analyzed by high resolution micro-Raman and scanning acoustic microscopy techniques ${ }^{36}$ that allow direct nondestructive, in situ detection of chemical structure and micromechanics, after which the same slabs are observed by field emission-scanning electron microscopy. ${ }^{29,37}$ These complementary analytical methods provide direct and comprehensive information regarding quality of the interface.

Dentin can be regarded as a biological composite of a collagen matrix which is highly filled with nanometer-sized apatite crystals. After extracting the mineral from the collagen fibrils, we can replace them with a resin to form a new composite of a resin matrix filled with a fibrous collagen. This new structure is a hybrid of resin and collagen. ${ }^{31,38}$ In this first FTIRI study of the dentin/adhesive interface, there is clear evidence that a structurally integrated collagen-polymer matrix is not formed at the interface between adhesive and caries-affected dentin. The chemistry of caries-affected dentin leads to greater variability and highly irregular composition along the length and breadth of the interface. The complexity of this clinically relevant substrate and its interface with dentin bonding agents cannot be readily conceived or appreciated without the global view provided by the FTIR images.

\section{Acknowledgments}

This investigation was supported in part by USPHS Research Grant Nos. DE 014392 (PS) and DE 015281 (YW) from the National Institute of Dental and Craniofacial Research, National Institutes of Health, Bethesda, MD 20892. Contribution from UMKC Center for Research on Interfacial Structure and Properties (UMKC-CRISP).

\section{References}

1. E. A. M. Kidd, F. Toffenetti, and I. A. Mjor, "Secondary caries," Int. Dent. J. 42, 127-138 (1992).

2. E. A. M. Kidd and D. Beighton, "Prediction of secondary caries around tooth-colored restorations: A clinical and microbiological study," J. Dent. Res. 75, 1942-1946 (1996).

3. C. J. Collins, R. W. Bryant, and K. L. V. Hodge, "A clinical evalutation of posterior composite resin restorations: 8-year findings," $J$. Dent. 26, 311-317 (1998).

4. A. R. Hunter, E. T. Treasue, and A. J. Hunter, "Increases in cavity volume associated with the removal of class 2 amalgam and composite restorations," Oper. Dent. 20, 2-6 (1995).

5. B. Van Meerbeek, M. Vargas, S. Inoue, Y. Yoshida, J. Perdigao, P. Lambrechts, and G. Vanherle, "Microscopy investigations. Techniques, results, limitations," Am. J. Dent. 13, 3-18 (2000).

6. H. Nordbo, J. Leirskar, and F. R. von der Fehr, "Saucer-shaped cavity preparations for posterior approximal resin composite restorations: observations up to 10 years," Quintessence Intl. 29, 5-11 (1998).

7. Adhesion: The Silent Revolution in Dentistry, J. F. Roulet and M. Degrange, Eds., Quintessence, Tokyo (2000).

8. G. Bergenholtz and C. F. Cox, "Bacterial leakage around dental restorations: Its effects on the dental pulp,' J. Oral Pathol. 11, 439-450 (1982).

9. R. H. Bullard, K. R. Leinfelder, and C. M. Russell, "Effect of coefficient of thermal expansion on microleakage," J. Am. Dent. Assoc. 116, 871-874 (1988)

10. T. J. Hilton, "Cavity sealers, liners, and based: Current philosophies and indications for use," Oper. Dent. 21, 134-146 (1996).

11. N. Nakabayashi and Y. Saimi, "Bonding to intact dentin," J. Dent. Res. 75, 1706-1715 (1996).

12. N. Nakabayashi and D. H. Pashley, Hybridization of Dental Hard Tissues, Quintessence, Tokyo (1998).

13. J. D. Eick, A. J. Gwinnet, D. H. Pashley, and S. J. Robinson, "Current concepts on adhesion to dentin," Crit. Rev. Oral Biol. Med. 8, 306-335 (1997).

14. A. J. Gwinnett, F. R. Tay, K. M. Pang, and S. H. Y. Wei, "Quantitative contribution of the collagen network in dentin hybridization," Am. J. Dent. 9, 140-144 (1996).

15. L. E. Tam and R. M. Pilliar, "Fracture surface characterization of dentin-bonded interfacial fracture toughness specimens," J. Dent. Res. 73, 607-619 (1994).

16. P. Spencer, Y. Wang, M. P. Walker, D. M. Wieliczka, and J. R. Swafford, "Interfacial chemistry of the dentin/adhesive bond," J. Dent. Res. 79, 1458-1463 (2000).

17. Y. Wang and P. Spencer, "Hybridization efficiency of the adhesive dentin interface with wet bonding," J. Dent. Res. 82, 141-145 (2003).

18. C. Harnirattisai, S. Inokoshi, H. Hosoda, and Y. Shimade, "Interfacial morphology of an adhesive composite resin and etched cariesaffected dentin," Oper. Dent. 17, 222-228 (1992).

19. M. Nakajima, H. Sano, M. F. Burrow, J. Tagami, M. Yoshiyama, S. Ebisu, B. Ciucchi, C. M. Russell, and D. H. Pashley, "Tensile bond strength and SEM evaluation of caries-affected dentin using dentin adhesives," J. Dent. Res. 74, 1679-1688 (1995).

20. M. Yoshiyama, R. M. Carvalho, H. Sano, J. A. Horner, P. D. Brewer, and D. H. Pashley, "Regional bond strengths of resins to human root dentine," J. Dent. 24, 435-442 (1996)

21. M. Yoshiyama, A. Urayama, T. Kimochi, T. Matsuo, and D. H. Pashley, "Comparison of conventional versus self-etching adhesive bonds to caries-affected dentin," Oper. Dent. 25, 163-169 (2000).

22. P. Spencer, Y. Wang, M. P. Walker, and J. R. Swafford, "Molecular structure of acid etched dentin smear layer-in situ study," J. Dent. Res. 80, 1802-1807 (2001).

23. Y. Wang and P. Spencer, "Studies on molecular structure of dentin smear debris by micro-Raman spectroscopy," J. Biomed. Mater. Res. 60, 300-308 (2002).

24. M. Nakajima, H. Sano, L. Zheng, J. Tagami, and D. H. Pashley, "Effect of moist versus dry bonding to normal versus caries-affected dentin with scotchbond multi-purpose plus," J. Dent. Res. 78, 12981303 (1999).

25. R. A. Farrell and R. L. McCauley, "On corneal transparency and its loss with swelling," J. Opt. Soc. Am. 66, 342-345 (1976).

26. Y. Wang and P. Spencer, "Quantifying adhesive penetration in adhesive/dentin interface using confocal Raman microspectroscopy," J. Biomed. Mater. Res. 59, 46-55 (2002). 
27. H. Sano, T. Yoshikawa, P. N. Percira, N. Kanemura, M. Morigami, J. Tagami, and D. H. Pashsley, "Long-term durability of dentin bonds made with a self-etching primer," J. Dent. Res. 78, 906-911 (1999).

28. P. Spencer and Y. Wang, "Adhesive phase separation at the dentin interface under wet bonding conditions," J. Biomed. Mater. Res. 62 , 447-456 (2002).

29. Y. Wang and P. Spencer, "Overestimating hybrid layer quality in polished adhesive/dentin interfaces," J. Biomed. Mater. Res. 68A. 735-746 (2004).

30. N. Nakabayashi, K. Kojima, and E. Masuhara, "The promotion of adhesion by the infiltration of monomers into tooth substrates," $J$. Biomed. Mater. Res. 16, 265-273 (1982).

31. N. Nakabayashi, "The hybrid layer: A resin-dentin composite," Proc. Finn Dent. Soc. 88, 321-329 (1992).

32. B. Van Meerbeek, A. Dhem, M. Goret-Nicaise, M. J. A. Braem, P. Lambrechts, and G. Vanherle, "Comparative SEM and TEM examination of the ultrastructure of the resin-dentin interdisffusion zone," J. Dent. Res. 72, 495-501 (1993).
33. M. Hashimoto, H. Ohno, M. Kaga, K. Endo, H. Sano, and H. Oguchi, "In vivo degradation of resin-dentin bonds in humans over 1 to 3 years," J. Dent. Res. 79, 1385-1391 (2000).

34. P. Spencer and J. R. Swafford, "Unprotected protein at the dentinadhesive interface," Quintessence Int. 30, 501-507 (1999).

35. Y. Wang and P. Spencer, "Effect of acid etching time and techniques on interfacial characteristics of the adhesive-dentin bond using differential staining," Eur. J. Oral Sci. 112, 293-299 (2004).

36. J. L. Katz, S. Bumrerraj, J. Dreyfuss, Y. Wang, and P. Spencer, "Micromechanics of the dentin/adhesive interface," J. Biomed. Mater. Res. 58, 366-371 (2001).

37. Y. Wang and P. Spencer, "Exploring the nature of acid-resistant hybrid layer with wet bonding," Oper. Dent. 29, 650-655 (2004).

38. N. Nakabayashi, M. Nakamura, and N. Yasuda, "Hybrid layer as a dentin bonding mechanism," J. Esthet Dent. 3, 133-138 (1991). 